\title{
Assimilation in networks promoting common pool resource conservation
}

Tuyen Tiet ( $\nabla$ tuyen.tiettong@gmail.com )

BETA, INRAE, University of Strasbourg https://orcid.org/0000-0001-9926-0248

\section{Research Article}

Keywords: Assimilation, Common-pool resource, Network, Social comparison

Posted Date: March 31st, 2021

DOI: https://doi.org/10.21203/rs.3.rs-310694/v1

License: (c) (i) This work is licensed under a Creative Commons Attribution 4.0 International License. Read Full License 


\title{
Assimilation in networks promoting common pool resource conservation
}

\author{
Tuyen Tiet $^{a *}$ \\ BETA, INRAE \& University of Strasbourg, France
}

This is a preliminary draft. Please do not cite or distribute without permission of the authors

March 10, 2021

\begin{abstract}
This study investigates how assimilation in social comparison ${ }^{1}$ (i.e., changing behaviors in order to fit in with a group) impacts individual behaviors in the extraction of a common pool resource in different network structures (i.e., empty network, star, circle, and complete network). Our results suggest that over-exploitation is more likely to happen when there is a presence of assimilation in comparison. However, it is possible to incentivize resource conservation since the assimilation effect on individual conservation behavior highly depends on the network structures. Thus, promoting assimilation to a centralized network or networks with fewer connections is a good way to encourage resource conservation. More particularly, in a decentralized network with fewer connections (e.g., a circle network), assimilation in social comparison (e.g., feedback on their behaviors and the average behaviors of their neighbors) could help to promote resource conservation. A centralized network is useful in diffusing information and promoting assimilation in comparison by incentivizing the resource conservation of the central agents.
\end{abstract}

Keywords: Assimilation; Common-pool resource; Network; Social comparison.

JEL Classification: D70; Q50.

\footnotetext{
${ }^{*}$ Corresponding author. Address: BETA, Université de Strasbourg, 61 avenue de la Forêt Noire, F-67000 Strasbourg, France; e-mail: tuyen.tiettong@gmail.com.

${ }^{1}$ Assimilation is when an individual can identify the similarities between the self and the other, while contrast refers to the heightened admiration/self-esteem one achieves after distancing oneself from others [12].
} 


\section{Introduction}

For centuries, our massive exploitation of common-pool resources (e.g., forests, oceans, fishing ponds, and atmosphere, etc.) has caused severe damages to the environment, such as ecological disturbances and pollution [17]. The theory of the tragedy of commons explained the massive exploitation by indicating that common-pool resources (henceforth CPRs) share the same "non-excludability" property with public goods (henceforth PGs). However, consumption of CPRs creates negative externality for all other people who share the CPRs, which is known as the "subtractability" [10]. The "subtractability" property means that an additional CPR consumption would decrease the available resources for others. As a result, it makes CPR management more complicated than a PG because it is hard to prevent people from subtracting units from a common pool.

Several existing literature supported the theory of Hardins (i.e., the theory of the tragedy of commons) by showing that when human behavior is assumed to be self-interest, open-access natural resources would undoubtedly lead to an over-exploitation problem $[4,20]$. It is because individuals would maximize their resource extraction by equalizing their marginal personal gains with the marginal cost of extraction. Moreover, since a common resource is scarce, each unit of resource extracted in a common pool would cause a negative externality for others. It would give rise to an inefficiently high level of extraction, which threatens the long-term sustainability of the common [24]. The Chilean abalone war, in which the competition between poachers and fishers in Chile to harvest abalone (the world's most valuable shellfish) leads to an armed conflict, is an example of the tragedy commons problem [9].

However, several studies have challenged Hardin's work by supporting a possibility of managing the commons $[16,18]$. In her study, Ostrom argued that with property rights and government regulations, one could achieve the successful management of CPRs [18]. ${ }^{2}$ In addition to the property rights and regulations, several existing literature suggested that social influences (e.g., norms, other-regarding preferences, social competition, etc.) are also effective ways to motivate the common resource conservation. One study indicated that individuals who have strong social preference would concern more about others' well-being, are willing to help others, and desires to uphold ethical norms (i.e., other-regarding preferences) [7]. ${ }^{3}$ For instance, in her book, Ostrom also cataloged several examples in which communities have successfully managed the CPRs without any help of the central government, from 16th-century Alpine shepherds managing grazing lands to the year of

\footnotetext{
${ }^{2}$ Elinor Ostrom got the Nobel prize for her work in 2009.

${ }^{3}$ Pro-social behavior have been studied widely in the context of public good game [11] and common pool resource game [22, 27] using the experimental data.
} 
1980s Japanese villages managing communal forests [16]. Additionally, some studies showed that people are willing to contribute to a public good if they know that others also contribute (i.e., conditional cooperation) $[1,7]$. In this sense, one may be motivated to extract less the CPRs if they observe others' conservation efforts. The others could stand for other people in the community or our close friends/neighbors (i.e., the ones we connect to).

Several studies have shown that networks could play an essential role in mitigating the over-extraction problem in recent years. One study indicated that when the populations dependent highly on the common resources, cooperation could also emerge and sustain if individuals are located in a network with close proximity and strong social ties $[6,8]$. Consequently, individuals often take others' decisions that they observed into considerations before making their decisions, especially the decisions of their close friends and neighbors [5, 26]. Some behavioral economic experiments suggested that information sharing through networks could restrain the resource extraction $[2,14]$. Notably, in their study, the authors found that fisheries in a network could share information with each other, thus reducing fish by-catch rate cooperatively [2]. Another study showed that cooperation in the CPR game and network structure plays a crucial role in reducing the aggregate extraction level of the CPR [14].

In this paper, we investigate how the social comparison in networks (i.e., ones are influenced by their close friends or neighbors' behaviors) affects individual behaviors in the context of the CPRs. In particular, we consider that individuals are located in a network, and there is assimilation in social comparison which ones imitate others' behaviors in their networks [15]. In the presence of a network, agents care more about their neighbors' behaviors since they are linked to each other rather than the strangers who are not in their network. Our objective is to explore how network structures and social comparison can lead to sustainable use and improve the management of the CPRs. More specifically, we aim to investigate how the assimilation in social comparison in different networks could impact individual behaviors in the common resource game.

To answer this question, we consider in our model that individuals care about the agents' behaviors that they connect to (i.e., friends or neighbors). In other words, each agent is located in a given network, and they compare their actions to their direct neighbors (i.e., social comparison in the network). We assume that the social comparison is assimilative, which means that one changes their behaviors to minimize the difference/distance between them and others, also known as "conformity". We consider the exogenous network to observe the network's causal effect on individual behavior as the network varies. We use the static framework of Nash equilibrium to examine the outcome of the CPR game under assumptions. Each agent in a network has to decide how much effort to put in extracting the common resource and at the same time take his or her direct neighborhood behaviors into account in order to maximize his or her personal utility. 
Our results suggest that over-exploitation is more likely to happen when there is a presence of assimilation in comparison. However, it is possible to incentivize resource conservation since the assimilation effect on individual conservation behavior highly depends on the network structures. More specifically, when the assimilation effect is low, a network with fewer connections (e.g., a circle network) promotes resource conservation. When the assimilation effect is high, only a centralized network (e.g., a star network) can help avoid over-exploitation. However, it is essential to promote resource conservation to the key players in the network to motivate individuals towards a more sustainable behavior.

The paper proceeds as follows. Section 2 studies the common resource game with social comparison using the general model. In Section 3, we compute the Nash equilibrium with the assumptions about the functional form of the production function and social comparison. In Section 4, we discuss the welfare analysis. Section 5 summarizes the results of our numerical analysis. The conclusion and discussion are discussed in Section 6.

\section{Common pool resource game}

\subsection{Setup}

Let consider that there are $N$ agents, a typical agent is denoted by $i$. Each agent $i$ has a set of neighbors which is denoted $N_{i}(g)$ (i.e., a network of $i$ ). Let $\mathbf{G}$ is an $N \times N$ adjacency matrix, its element $g_{i j}$ represents the relationship between $i$ and $j$. In particular, if $i$ is linked to $j$, then we have $g_{i j}=1$ for $j \in N_{i}(g)$ and if $i$ is not linked to $j$, then we have $g_{i j}=0$ for $j \notin N_{i}(g)$. We suppose that the network is undirected which requires that if $g_{i j}=1$ then $g_{j i}=1$, which means that if $i$ is neighbor of $j$, then $j$ is also neighbor of $i^{4}$. The network of $i$ is a set of nodes that is linked to $i: N_{i}(g)=\left\{j \backslash i: g_{i j}=1\right\}$ or $N_{i}(g)=\{j \mid i j \in g\}$. Let $k_{i}=\left|N_{i}(g)\right|$ be agent $i$ 's number of neighbors or agent $i$ 's centrality. ${ }^{5}$

An agent $i$ will face a decision problem of how to optimally allocate his/her effort $x_{i}$ to extract the resources when taking their neighbors' behaviors into account. We assume that agent $i$ 's effort is bounded $x_{i} \in[0, \bar{x}]$, where $\bar{x}$ is the effort capacity. According to "substractability" property of

\footnotetext{
${ }^{4}$ For instance, there are 3 agents in the network, $N=\{1,2,3\}$ and the adjacency matrix $\mathbf{G}$ is given by

$$
\mathbf{G}=\left[\begin{array}{lll}
0 & 1 & 1 \\
1 & 0 & 1 \\
1 & 1 & 0
\end{array}\right]
$$

This matrix suggests that there is a circle network of 3 agents in which agent 1 links to agent 2 and 3 , agent 2 links to 1 and 3 , and 3 links to 1 and 2 .

${ }^{5}$ Centrality of a node is the number of agents that are connected to the agent $i[23]$.
} 
the CRP, the share of common resources extracted by $i$ is $\frac{x_{i}}{x_{i}+x_{-i}}$, which is the proportion of effort that $i$ make in extracting the resources with respect to the total sum of all agents' efforts (ref?). We assume that total effort function $f(X)$ is concave, in which $f(0)=0, f(X)>0$ if $X>0$, $f^{\prime}(X)>0$ and $f^{\prime \prime}(X) \leq 0$, where the total effort $X=\sum_{i} x_{i}=x_{i}+x_{-i}$.

Each agent has a network of neighbors in which (s)he could observe others' behaviors and compare his/her behavior with others. We denote the difference between agent $i$ and his/her neighbors by $I\left(x_{i}, \mathbf{x}_{N_{i}(g)} ; \delta_{i}\right)$, where $\mathbf{x}_{N_{i}(g)}$ is a set of $i$ 's neighborhood efforts, and $\delta_{i}$ is the comparison parameter which is heterogeneous across agents and takes negative values (i.e., $\delta_{i}<0$ ). The negative $\delta_{i}<0$ refers to the assimilation in comparison (i.e., conformity), which means that agents change their behaviors to fit in their groups. We do not explore the possibility of the contrast in comparison (i.e., $\delta_{i}>0$ ) in this study because no agent is willing to differentiate with others by putting less effort in extracting the common resource (since there is a "substractability" property of the common). On the other hand, because of the contrast in comparison, all agents would put as much effort to extract the resource, leading to the over-exploitation of the CPRs.

We consider that there exists an ability parameter $\eta_{i}>0$ such that agent $i$ gains an amount $\eta_{i} x_{i}$ for each unit of $x_{i}$ increasing. In other words, agents are heterogeneous in their ability (i.e., $\eta_{i} \neq \eta_{j}$ ), and an agent with a higher ability (higher value of $\eta$ ) would be able to gain a higher benefit from each unit of extracted resource. There exist two types of heterogeneity in our model: idiosyncratic heterogeneity and peer heterogeneity. The idiosyncratic heterogeneity captures the fact that agents differ in their ability in extracting the common resource [19]. The peer heterogeneity comes from the different network structure, different comparison parameters, and agents with different reference groups in the social comparison. For example, with a network of $N$ agents, the central agents in the star network have the same number of direct neighbors as agents in the complete network. These two agents have different reference groups because each neighbor of the agent in the complete network has $N-1$ neighbors, while each neighbor of the agent in the center of the star network has only 1 neighbor. The peer heterogeneity also comes from the difference in comparison parameter, which means that agents care differently about their neighbors' actions.

\subsection{Model}

In the spirit of [24], we extend the model of the CPR game by taking the role of network and social comparison into account. ${ }^{6}$ For a given network structure, agent $i$ 's utility function is written as

\footnotetext{
${ }^{6}$ We also follow the paper of [19], which studied the social comparison in crime, for the idea of how to model the social comparison in the network.
} 
follows:

$$
\begin{array}{ll} 
& U_{i}\left(x_{i}\right)=\underbrace{f(X) \frac{x_{i}}{X}}_{\text {benefit }}-\underbrace{\gamma x_{i}^{2}}_{\text {cost }}+\underbrace{\eta_{i} x_{i}}_{\text {ability }}+\underbrace{I\left(x_{i}, \mathbf{x}_{N_{i}(g)} ; \delta_{i}\right)}_{\text {social comparison }} \\
\text { s.t } & x_{i} \leq \bar{x}, \quad x_{i} \geq 0
\end{array}
$$

where, $X=x_{i}+\sum_{j \neq i} x_{j}$ is the total effort and $\bar{x}$ is the effort's capacity.

From the optimization problem 1, we have the following Lagrangian.

$$
\mathcal{L}\left(x_{i}\right)=f(X) \frac{x_{i}}{X}-\gamma x_{i}^{2}+\eta_{i} x_{i}+I\left(x_{i}, \mathbf{x}_{N_{i}(g)} ; \delta_{i}\right)-\mu_{1}\left(x_{i}-\bar{x}\right)+\mu_{2} x_{i}
$$

\section{The Karush-Kuhn-Tucker conditions}

In the spirit of the Karush-Kuhn-Tucker (KKT) theorem, we have

$$
\begin{aligned}
\frac{\partial \mathcal{L}\left(x_{i}\right)}{\partial x_{i}} & =0 \\
\mu_{1}\left(x_{i}-\bar{x}\right) & =0 \\
\mu_{2} x_{i} & =0 \\
\mu_{m} & \geq 0, \quad m=\{1,2\} \\
x_{i} & \leq \bar{x} \\
x_{i} & \geq 0
\end{aligned}
$$

Concerning the complementary slackness condition 5 which is $\mu_{2} x_{i}=0$, we consider following cases.

Case 1. The condition $x_{i} \geq 0$ is binding. In this case, we have $x_{i}=0$. Then, the agent $i$ 's utility equals to

$$
U_{i}(0)=I\left(0, \mathbf{x}_{N_{i}(g)} ; \delta_{i}\right)
$$

We observe that $U_{i}(0) \leq 0$ since there is an assimilation in comparison (i.e., $\delta_{i}<0$ ). At the equilibrium, $\underline{x}^{c}=0$ for all $i$ and thus $U_{i}(0)=I\left(0, \mathbf{x}_{N_{i}(g)} ; \delta_{i}\right)=0, \forall \delta_{i}<0$.

Case 2. The condition $x_{i} \geq 0$ is not binding. In this case, in order for the slackness condition 5 to be satisfied, we need $\mu_{2}=0$. Thus, we have the following Lagrangian.

$$
\mathcal{L}\left(x_{i}\right)=f(X) \frac{x_{i}}{X}-\gamma x_{i}^{2}+\eta_{i} x_{i}+I\left(x_{i}, \mathbf{x}_{N_{i}(g)} ; \delta_{i}\right)-\mu_{1}\left(x_{i}-\bar{x}\right)
$$

Now, concerning the complementary slackness conditions 4 which is $\mu_{1}\left(x_{i}-\bar{x}\right)=0$, by the same logic, we also consider the following 2 cases.

Case 2.a. Constraint $x_{i} \leq \bar{x}$ is binding which is $x_{i}=\bar{x}$. In this case, we have a corner solution 
$\bar{x}^{c}=\bar{x}$.

Case 2.b. Constraint $x_{i} \leq \bar{x}$ is not binding. Thus, we need $\mu_{1}=0$ in order for the complementary slackness conditions 4 to be satisfied. This is a case that agent's effort cannot be equal to zero or reach the maximum effort's capacity, i.e., $x_{i} \in(0, \bar{x})$. Then, we can write the KKT conditions as follows:

$$
\frac{\partial \mathcal{L}\left(x_{i}\right)}{\partial x_{i}}=f^{\prime}(X) \frac{x_{i}}{X}+f(X) \frac{x_{-i}}{X^{2}}-2 \gamma x_{i}+\eta_{i}+\frac{\partial I_{i}\left(x_{i}\right)}{\partial x_{i}}=0
$$

where, $I_{i}\left(x_{i}\right) \equiv I\left(x_{i}, \mathbf{x}_{N_{i}(g)} ; \delta_{i}\right)$. Thus, this KKT condition is a necessary condition for the maximization problem.

\section{Sufficient condition of optimality}

In order to ensure the existence of a maximum, we need $\frac{\partial^{2} U_{i}}{\partial x_{i}^{2}} \leq 0$. Following the second order sufficient condition, we have the following inequality ${ }^{7}$

$$
\frac{\partial U_{i}^{2}}{\partial^{2} x_{i}}=\frac{1}{X^{2}}\left\{f^{\prime \prime}(X) X x_{i}+2 x_{-i}\left(f^{\prime}(X)-\frac{f(X)}{X}\right)\right\}-2 \gamma+\frac{\partial^{2} I_{i}\left(x_{i}\right)}{\partial x_{i}^{2}}
$$

Since $x_{i}, x_{-i}>0$, we have $f^{\prime \prime}(X)<0$ and $f^{\prime}(X) X-f(X)<0^{8}$. The condition $\frac{\partial^{2} U_{i}}{\partial x_{i}^{2}} \leq 0$ if we have $\frac{\partial^{2} I_{i}\left(x_{i}\right)}{\partial x_{i}^{2}} \leq 0$. Thus, the condition $\frac{\partial^{2} U_{i}}{\partial x_{i}^{2}} \leq 0$ will hold in the case of assimilation in comparison if $\frac{\partial^{2} I_{i}\left(x_{i}\right)}{\partial x_{i}^{2}} \leq 0$ holds.

Therefore, the sufficient condition leads us to the following Lemma 1.

Lemma 1. The optimization problem always has a maxima in the case of assimilation in comparison (i.e., $\left.\delta_{i}<0\right)$ if $\frac{\partial^{2} I_{i}\left(x_{i}\right)}{\partial x_{i}^{2}} \leq 0$.

In the case of assimilation in comparison, agents mitigate others' behaviors in their network. This leads to the fact that $\frac{\partial I_{i}\left(x_{i}\right)}{\partial x_{i}}<0$ for $x_{i} \neq \mathbf{x}_{N_{i}(g)}$ (i.e., $x_{i}$ is different from the standard neighborhood extraction). Thus, in order to ensure a maximum, we need $\frac{\partial^{2} I_{i}\left(x_{i}\right)}{\partial x_{i}^{2}} \leq 0$ which means that a more difference in extraction compared to the standard neighborhood extraction, more agents suffer from the benefit loss form the social comparison. Therefore, we need the above condition holds in order for the optimization problem to have a maximum, i.e., an interior equilibrium solution). If not, all agents would choose to extract the resource with the maximum effort's capacity. Thus, it indeed leads to an over-exploitation problem of CPR.

\footnotetext{
${ }^{7}$ See the proof in Appendix.

${ }^{8} f(x)$ is a concave function
} 


\section{Nash equilibrium level of effort}

To calculate a definitive solution for the equilibrium level of effort and welfare analysis in the next section, we make some assumptions about the functional forms of the total effort function and social comparison.

In the spirit of [27] in limit-access common pool resource, we let $f(X)=a X-b X^{2}$ be the effort function for the total effort $X$, where $a \geq 0$ and $b \geq 0$. We can check that the function $f(X)$ satisfies our assumptions.

$$
f(X) \geq 0 \text { if } X \leq \frac{a}{b}, \quad \quad f^{\prime}(X)=a-2 b X \geq 0 \text { if } X \leq \frac{a}{2 b}, \quad f^{\prime \prime}(X)=-2 b \leq 0 .
$$

Let $\bar{x}_{N_{i}(g)}$ be the reference level of effort that the agent $i$ compared to. We consider the social comparison as a difference between $\bar{x}_{N_{i}(g)}$ and $x_{i}$, such that

$$
I\left(x_{i}, \bar{x}_{N_{i}(g)} ; \delta_{i}\right)=\delta_{i}\left(x_{i}-\bar{x}_{N_{i}(g)}\right)^{2} .
$$

It should be noted that the reference level could be the neighbor's average, maximum or minimum level of effort. We suppose that the reference level is the direct neighbors' average level of effort. Thus, we have

$$
\bar{x}_{N_{i}(g)}=\frac{1}{k_{i}} \sum_{j}^{N} g_{i j} x_{j},
$$

where, $k_{i}$ is number of agent $i$ 's direct neighbors.

In the case of assimilation in comparison, we have $\delta_{i}<0$ which means that agents would suffer a negative impact of the social comparison for each unit of effort higher than the reference level. We could check that

$$
\frac{\partial I_{i}\left(x_{i}\right)}{\partial x_{i}}=2 \delta_{i}\left(x_{i}-\bar{x}_{N_{i}(g)}\right) \leq 0 \text { if } x_{i}-\bar{x}_{N_{i}(g)} \geq 0 .
$$

And we have also that

$$
I_{x x}=\frac{\partial^{2} I_{i}\left(x_{i}\right)}{\partial x_{i}^{2}}=2 \delta_{i}<0
$$

This result suggests that with a more increasing extraction of a common resource, the agent suffers a negative impact from the comparison. Thus, from Lemma 1, we could confirm that the sufficient condition for optimality always holds in the case of assimilation in comparison.

In this study, we will focus on different scenarios in which $\delta_{i}<0$ in order to study how the different value of $\delta_{i}$ and network structure can help impacts on agents' behaviors in extracting/consuming the common goods. 


\subsection{Equilibrium}

We now examine the model's outcome with social comparison under the assumption that agents are heterogeneous in the social comparison (i.e., agents care differently about their direct neighbors' actions).

Let $\mathbf{x}=\left(x_{1}, x_{2}, x_{3}, \ldots, x_{N}\right)$ and thus $\mathbf{x}$ is the solution of the following maximization problem

$$
\max _{x_{i}} U_{i}(\mathbf{x})=\max _{x_{i}}\left\{f(X) \frac{x_{i}}{X}-\gamma x_{i}^{2}+\eta_{i} x_{i}+\delta_{i}\left(x_{i}-\frac{1}{k_{i}} \sum_{j}^{N} g_{i j} x_{j}\right)^{2}\right\}
$$

s.t $\quad x_{i} \leq \bar{x}, \quad x_{i} \geq 0$

From the optimization problem 11, we have the following Lagrangian

$$
\mathcal{L}(\mathbf{x})=(a-b X) x_{i}-\gamma x_{i}^{2}+\eta_{i} x_{i}+\delta_{i}\left(x_{i}-\frac{1}{k_{i}} \sum_{j}^{N} g_{i j} x_{j}\right)^{2}-\mu_{1}\left(x_{i}-\bar{x}\right)+\mu_{2} x_{i}
$$

We have the F.O.C of the Lagrangian as follows.

$$
\frac{\partial \mathcal{L}(\mathbf{x})}{\partial x_{i}}=a-b\left(X+x_{i}\right)-2 \gamma x_{i}+\eta_{i}+2 \delta_{i}\left(x_{i}-\frac{1}{k_{i}} \sum_{j}^{N} g_{i j} x_{j}\right)-\mu_{1}+\mu_{2}=0
$$

Recall the KKT conditions, we can check whether all the conditions are satisfied.

Case 1. We have the corner solution $\underline{x}^{c}=0$ and the utility $U_{i}(0)=0$. In this case, agent make no effort in extracting the resource and thus there will be no benefit gained at this lower corner solution.

Case 2. We have $x_{i}>0, \forall i$. Thus, by the complementary slackness condition 5 and 4 , we have 2 following cases.

Case 2.a. We have the constraint $x_{i} \leq \bar{x}$ is binding. Then, by the slackness condition, we have $x_{i}=\bar{x}$ and $\mu_{1} \geq 0$. Thus, we have

$$
\frac{\partial \mathcal{L}(\mathbf{x})}{\partial x_{i}}=a-b\left(X+x_{i}\right)-2 \gamma x_{i}+\eta_{i}+2 \delta_{i}\left(x_{i}-\frac{1}{k_{i}} \sum_{j}^{N} g_{i j} x_{j}\right)-\mu_{1}=0
$$

From the slackness condition, we have $x_{i}=\bar{x}$. Thus, at the equilibrium, we have $\bar{x}^{c}=\bar{x}$ in which all agents extract the resource at the maximum effort's capacity. We have a upper corner solutions, such that

$$
\bar{x}^{c}=\bar{x}, \quad \text { and } \mu_{1, i}=a+\eta_{i}-[b(N+1)+2 \gamma] \bar{x} .
$$

It should be noted that this upper corner solution will exist if and only if we have the condition $\mu_{1, i}=a+\eta_{i}-[b(N+1)+2 \gamma] \bar{x} \geq 0$ to be satisfied. 
Case 2.b. In this case, we have $\mu_{1}=0$. Let $x^{*}$ be the interior equilibrium solution. From the KKT condition, we have

$$
x_{i}=\frac{1}{2\left(b+\gamma-\delta_{i}\right)}\left(a+\eta_{i}-b \sum_{j \neq i}^{N} x_{j}-2 \delta_{i} \frac{1}{k_{i}} \sum_{j}^{N} g_{i j} x_{j}\right)
$$

\section{Symmetric case}

Before going the general case in which the agents are heterogeneous in their social comparison, we first discuss the situation that all agents are identical. In this case, we have $\eta_{i}=\eta$ which means that all agents are identical and thus has a same ex-ante heterogeneity. From Equation (13), we have the first proposition as follows:

Proposition 1. When $\eta_{i}=\eta$, we have $x^{*}=x_{i}^{*}=x_{j}^{*}$ at the equilibrium

$$
x^{*}=\frac{a+\eta}{(N+1) b+2 \gamma}
$$

When agents are identical, a higher ability agent (i.e., agent has a higher value of $\eta$ ) will put more effort into extracting the common resource. On the other hand, increasing extraction cost by either increasing the value of $\gamma$ could incentivize agents to reduce their extraction efforts. Another way to reduce extraction effort is by reducing the parameter $a$ or/and increasing the parameter $b .^{9}$ Therefore, in order to incentivize the common resource conservation, we should increase the cost of resource extraction and reduce individual heterogeneity as well as reduce the profitability of the effort function [21].

It should be noted that when agents are homogeneous in their ability, we observe the social comparison and network structure do not have any influence on an agent's behavior. Consequently, all agents are identical, and thus they would have the same equilibrium solution. Therefore, the social comparison does not play any role at the equilibrium because agents do not have to care about the difference between them and their direct neighbors.

\section{General case}

In this case, we have $\eta_{i} \neq \eta_{j} \neq \eta, \forall i \neq j$ which suggests that agents are heterogeneous in their ability in extracting the resource. Since agents are not identical because of the idiosyncratic heterogeneity

\footnotetext{
${ }^{9}$ Recall that $a$ is a parameter of the effort function $f(X)=a X-b X^{2}$. With a lower value of $a$ and a higher value of $b$, fewer benefit gains from extracting the common resource.
} 
and peer heterogeneity (they are also different because of the different network structure and thus have the different reference group in the social comparison), each agent would have a different equilibrium effort at the equilibrium.

Let $\mathrm{x}^{*}$ is a $N \times 1$ column matrix (vector) of the each agent equilibrium extraction effort $x_{1}^{*}, x_{2}^{*}, x_{3}^{*}, \ldots, x_{N}^{*}$. We could simply rewrite Equation 13 as follow.

$$
\mathbf{x}^{*}=\frac{1}{2(b+\gamma)}\left(\iota+\alpha \boldsymbol{\Phi}_{1} \mathbf{x}^{*}\right)
$$

where, $\alpha=\frac{1}{2(b+\gamma)}$, the $N \times N$ matrix $\boldsymbol{\Phi}_{1}$ is

$$
\boldsymbol{\Phi}_{1}=2 \boldsymbol{\delta}(\mathbf{I}-\mathbf{K G})-b \boldsymbol{\Delta}
$$

in which, $\iota$ is a $N \times 1$ column matrix which each element equals to $a+\eta_{i}$, matrix $\boldsymbol{\delta}$ is a $N \times N$ matrix of the comparison parameter such that

$$
\boldsymbol{\delta}=\left[\begin{array}{cccc}
\delta_{1} & 0 & \ldots & 0 \\
0 & \delta_{2} & \ldots & 0 \\
\ldots & \ldots & \ldots & \ldots \\
0 & 0 & \ldots & \delta_{N}
\end{array}\right],
$$

K is a $N \times N$ matrix of the number of network connections such that

$$
\mathbf{K}=\left[\begin{array}{cccc}
\frac{1}{k_{1}} & 0 & \ldots & 0 \\
0 & \frac{1}{k_{2}} & \ldots & 0 \\
\ldots & \ldots & \ldots & \ldots \\
0 & 0 & \ldots & \frac{1}{k_{N}}
\end{array}\right],
$$

$\Delta$ is a $N \times N$ matrix such that

$$
\boldsymbol{\Delta}=\left[\begin{array}{cccc}
0 & 1 & \ldots & 1 \\
1 & 0 & \ldots & 1 \\
\ldots & \ldots & \ldots & \ldots \\
1 & 1 & \ldots & 0
\end{array}\right],
$$

and the $N \times N$ matrix $\mathbf{G}$ which is known as the adjacent matrix.

Proposition 2. Let $\mathbf{x}^{*}=\left(x_{1}^{*}, x_{2}^{*}, x_{3}^{*}, \ldots, x_{N}^{*}\right)$ be the equilibrium level of extraction. If the following matrix $\left(\mathbf{I}-\alpha \mathbf{\Phi}_{1}\right)$ is invertible, then the CPR game has an interior equilibrium solution which equals to

$$
\mathbf{x}^{*}=\frac{1}{2(b+\gamma)}\left(\mathbf{I}-\alpha \mathbf{\Phi}_{1}\right)^{-1} \iota
$$


where, $\boldsymbol{\Phi}_{1}=2 \boldsymbol{\delta}(\mathbf{I}-\mathbf{K G})-b \boldsymbol{\Delta}$.

From this closed-form solution, one can calculate the interior equilibrium level of effort for a given network structure. We observe that the equilibrium solution depends positively on the agent's ability and negatively on extraction cost. We can also observe that a higher comparison parameter results in a lower extraction of the common resource in the case of contrast in comparison $\left(\delta_{i}<0\right)$. In the case of assimilation in comparison, a more increase in the comparison parameter, a higher probability that there is an over-exploitation of the common. This interior solution also suggests that the network structure would play a key role in encouraging the conservation of the common resource. A network with more connections would have a lower level of extraction than the one with fewer connections.

\subsection{Over-exploitation of CPR}

As discussed previously, we have three equilibrium solutions: $\underline{x}^{c}=0, \bar{x}^{c}=\bar{x}$ and $x^{*}$ which is a solution of the following matrix $\mathbf{x}^{*}=\frac{1}{2(b+\gamma)}\left(\mathbf{I}-\alpha \mathbf{\Phi}_{1}\right)^{-1} \boldsymbol{\iota}$. But, we will focus our discussion on the upper corner and the interior equilibrium solution since the lower corner solution in which all agents have zero utility is not interesting to study. By assuming that $x_{i}>0, \forall i$, agents could either decide to make an effort which equals to the maximum effort capacity $\bar{x}$ or the interior equilibrium $x^{*}$. It should be important to note that $x^{*}<\bar{x}$ and thus from a policy-maker's point of view, we want to avoid the solution $\bar{x}^{c}=\bar{x}$ which leads to the over-exploitation of the common.

In fact, individual agent $i$ would refer $x_{i}^{*}$ to $\bar{x}_{i}^{c}$ if and only if

$$
U_{i}\left(\mathbf{x}_{i}^{*}\right) \geq U_{i}\left(\overline{\mathbf{x}}_{i}^{c}\right)
$$

Since we have $\bar{x}_{i}-\bar{x}_{N_{i}(g)}=0$, the previous condition is equivalent to

$$
f\left(X^{*}\right) \frac{x_{i}^{*}}{X^{*}}-\gamma x_{i}^{* 2}+\eta_{i} x_{i}^{*}+\delta_{i}\left(x_{i}^{*}-\bar{x}_{N_{i}(g)}^{*}\right)^{2} \geq f(\bar{X}) \frac{\bar{x}_{i}^{c}}{\bar{X}}-\gamma\left(\bar{x}_{i}^{c}\right)^{2}+\eta_{i} \bar{x}_{i}^{c},
$$

where, $\bar{X}=\sum_{i} \bar{x}_{i}^{c}$ and $X^{*}=\sum_{i} x_{i}^{*}$.

\section{Symmetric case}

In the symmetric case, we have $x_{i}^{*}=x_{j}^{*}$ and thus $x_{i}^{*}-\bar{x}_{N_{i}(g)}^{*}=0$. Then, the inequality (17) is equivalent to

$$
f\left(X^{*}\right) \frac{x^{*}}{X^{*}}-\gamma x^{* 2}+\eta_{i} x^{*} \geq f(\bar{X}) \frac{\bar{x}_{i}^{c}}{\bar{X}}-\gamma\left(\bar{x}_{i}^{c}\right)^{2}+\eta_{i} \bar{x}_{i}^{c}
$$

Since $x^{*}-\bar{x} \leq 0$, with some simply algebra (see the proof in the Appendix), we have

$$
\gamma \geq \frac{a+\eta-N b\left(x^{*}+\bar{x}\right)}{x^{*}+\bar{x}} \equiv \underline{\gamma} .
$$


Therefore, in the symmetric case, in order to avoid the over-exploitation problem, we need to ensure a sufficiently high extraction cost $\gamma \geq \bar{\gamma} \equiv \frac{a+\eta-N b\left(x^{*}+\bar{x}\right)}{x^{*}+\bar{x}}$. From this condition, we could observe that the over-exploitation would not be easily bypassed unless we have either a sufficiently high extraction cost $\gamma$ or low benefit gained from CPR extraction (i.e., if there is a low parameter value of $a$ or $\eta$ ). It should be noted that increasing $N$ would help to reduce $\underline{\gamma}$, but this would not be a good solution because a high value $N$ (i.e., there are too many agents) would lead to the situation of excess demand because of the resource scarcity.

\section{General case}

Let $\theta_{i}^{*}=x_{i}^{*}-\bar{x}_{N_{i}(g)}^{*}$ be the difference between the equilibrium of $i$ and the average equilibrium extraction of his/her direct neighbors. In some cases (for example, symmetric network such as a complete and circle network in which agents have a same number of network connection), the $\theta^{*}$ equals to zero because all agents are identical. However, generally, we assume that $\theta_{i}^{*} \geq 0, \forall i$. We have the condition (17) equivalent to

$$
\gamma \geq \frac{a+\eta_{i}}{x_{i}^{*}+\bar{x}}+\frac{\delta_{i} \theta_{i}^{* 2}-b\left(X^{*} x_{i}^{*}-N \bar{x}\right)}{\left(x_{i}^{*}-\bar{x}\right)\left(x_{i}^{*}+\bar{x}\right)} \equiv \underline{\gamma}_{i} .
$$

Therefore, this leads us to the following proposition.

Proposition 3. An agent $i$ would refer the interior equilibrium $x_{i}^{*}$ which is a solution of the matrix $\mathbf{x}^{*}=\frac{1}{2(b+\gamma)}\left(\mathbf{I}-\alpha \mathbf{\Phi}_{1}\right)^{-1} \iota$ to the upper corner equilibrium $\bar{x}_{i}^{c}=\bar{x}$ if $\gamma \geq \underline{\gamma}_{i}$ with $\underline{\gamma}_{i}=\frac{a+\eta_{i}}{x_{i}^{*}+\bar{x}}+\frac{\delta_{i} \theta_{i}^{* 2}-b\left(X^{*} x_{i}^{*}-N \bar{x}\right)}{\left(x_{i}^{*}-\bar{x}\right)\left(x_{i}^{*}+\bar{x}\right)}$.

This proportion suggests that with $\gamma$ is sufficiently large, which means that the cost of extraction has to be sufficiently high, we can avoid the over-exploitation problem of the common because the agent always refers the interior solution $x^{*}$ to the corner one $\bar{x}^{c}=\bar{x}$. According to the result of Proposition 3, it is interesting to separate our discussions into two different parts: the impact of neighborhood decisions $\left(\theta_{i}^{*}\right)$ and the impact of social comparison $\left(\delta_{i}\right)$.

Firstly, by taking the derivative of $\underline{\gamma}_{i}$ with respect to $\theta_{i}^{*}$, we have $\frac{\partial \underline{\gamma}_{i}}{\partial \theta_{i}^{*}}=\frac{2 \delta_{i} \theta_{i}^{*}}{\left(x_{i}^{*}-\bar{x}\right)\left(x_{i}^{*}+\bar{x}\right)}$, where $\theta_{i}^{*}=x_{i}^{*}-\bar{x}_{N_{i}(g)}^{*}$. We could observe that a positive or negative of $\frac{\partial \underline{\gamma}_{i}}{\partial \theta_{i}^{*}}$ depending on 2 different situations: (i) $\theta_{i}^{*}=0$ and (ii) $\theta_{i}^{*} \neq 0$.

(i) In the first case in which we have $\theta_{i}^{*}=0$, the previous condition is equivalent to

$$
\gamma \geq \frac{a+\eta-N b\left(x^{*}+\bar{x}\right)}{x^{*}+\bar{x}}
$$

It should be noted that in the case that $\theta_{i}^{*}$ closes to zero means that there is no significant difference between the equilibrium extraction of agent $i$ and his/her direct neighbors. This result is similar 
to the symmetric case as discussed previously. Thus, the over-exploitation problem will be more likely to happen with $\theta_{i}^{*}=0$.

(ii) In the case $\theta_{i}^{*} \neq 0$, we would have

$$
\frac{\partial \underline{\gamma}_{i}}{\partial \theta_{i}^{*}}=\frac{2 \delta_{i} \theta_{i}^{*}}{\left(x_{i}^{*}-\bar{x}\right)\left(x_{i}^{*}+\bar{x}\right)}
$$

We observe that $\frac{\partial \underline{\gamma}_{i}}{\partial \theta_{i}^{*}}<0$ when $\theta_{i}^{*}=x_{i}^{*}-\bar{x}_{N_{i}(g)}^{*}<0$. This means that as $\theta_{i}^{*} \rightarrow 0$, we have $\underline{\gamma}_{i}$ increases. As a result, the increasing assimilation effect of the social comparison leads to the more serious over-extraction of the common resource. In order to bypass the over-exploitation problem, we $\theta_{i}^{*}=x_{i}^{*}-\bar{x}_{N_{i}(g)}^{*}>0$ which means that agents extract more resource than their direct neighbors at the equilibrium.

Secondly, by taking the derivative of $\underline{\gamma}_{i}$ with respect to $\delta_{i}$, we have $\frac{\partial \underline{\gamma}_{i}}{\partial \delta_{i}}=\frac{\theta_{i}^{* 2}}{\left(x_{i}^{*}-\bar{x}\right)\left(x_{i}^{*}+\bar{x}\right)}$. Obviously, we have $\frac{\partial \underline{\gamma}_{i}}{\partial \delta_{i}} \leq 0$ because $x_{i}^{*}<\bar{x}, \forall i$. However, since there is an assimilation in comparison, we would also have $\theta_{i}^{*} \rightarrow 0$ as $\delta_{i}$ increases. Therefore, a more increasing in the comparison parameter, a less likely we reach the over-exploitation problem of the CPR if we have $\theta_{i}^{*}>0$.

Therefore, maintaining a sufficiently high extraction cost of the CPR will not be the only solution to avoid the over-exploitation problem. Encouraging the assimilation in social comparison could also help conserve the CPRs if there is $\theta_{i}^{*}>0$ such that $\underline{\gamma}_{i}$ decreases as $\delta_{i}$ increases.

\section{Welfare analysis}

Let consider that the social planner is an utilitarian so he would maximize the total agent's payoff.

$$
\begin{gathered}
\max _{x_{i}, x_{2}, \ldots, x_{N}} \sum_{i} U_{i}(\mathbf{x})=\max _{x_{i}, x_{2}, \ldots, x_{N}} \sum_{i}^{N}\left\{f(X) \frac{x_{i}}{X}-\gamma x_{i}^{2}+\eta_{i} x_{i}+\delta_{i}\left(x_{i}-\frac{1}{k_{i}} \sum_{j}^{N} g_{i j} x_{j}\right)^{2}\right\} \\
\text { s.t } 0 \leq x_{i} \leq \bar{x} .
\end{gathered}
$$

By taking the F.O.C of Equation (18) with respect to $x_{i}$ for all $i=1, \ldots N$, we have

$$
a-2 b \sum_{i}^{N} x_{i}-2 \gamma x_{i}+\eta_{i}+2 \delta_{i}\left(x_{i}-\frac{1}{k_{i}} \sum_{j}^{N} g_{i j} x_{j}\right)=0
$$

Then, we obtain

$$
x_{i}=\frac{a+\eta_{i}-\frac{2 \delta_{i}}{k_{i}} \sum_{j} g_{i j} x_{j}}{2\left(N b+\gamma-\delta_{i}\right)}
$$

In the case of symmetric agents (i.e., $\eta_{i}=\eta, \forall i$ ), we have the social optimal effort $\hat{x}$ which equals to

$$
\hat{x}=\frac{a+\eta}{2(N b+\gamma)}
$$


In general, we have heterogeneous agents in which $\delta_{i} \neq \delta_{j} \neq \delta$. Let $\hat{\mathbf{x}}$ be the $N \times 1$ matrix of the social optimal extraction effort. In the matrix formula, we have

$$
\hat{\mathbf{x}}=\frac{1}{2(b+\gamma)} \iota+2 \alpha \Phi_{2} \hat{\mathbf{x}}
$$

where, $\alpha=\frac{1}{2(b+\gamma)}$, the $N \times N$ matrix $\boldsymbol{\Phi}_{2}$ such that

$$
\mathbf{\Phi}_{2}=\boldsymbol{\delta}(\mathbf{I}-\mathbf{K G})-b \boldsymbol{\Delta}
$$

and the definition of other matrices are similar to the previous session. Thus, if the following matrix $\left(\mathbf{I}-2 \alpha \boldsymbol{\Phi}_{2}\right)$ is invertible, then we have the social optimal extraction effort as follows:

$$
\hat{\mathbf{x}}=\frac{1}{2(b+\gamma)}\left(\mathbf{I}-2 \alpha \boldsymbol{\Phi}_{2}\right)^{-1} \iota
$$

\section{Efficiency}

In the symmetric case, by comparing the interior equilibrium effort $x^{*}$ (in Equation (14)) and the social optimal effort $\hat{\mathbf{x}}$ (in Equation (19)), we observe that for any $N>1$, we always have $\hat{x}<x^{*}$. We can also check that the social optimal effort is socially efficient because $U(\hat{x}) \geq U\left(x^{*}\right)$.

$$
\begin{aligned}
U(\hat{x}) & \geq U\left(x^{*}\right) \\
\Leftrightarrow \quad(a-b \hat{X}) \hat{x}-\gamma \hat{x}^{2}+\eta \hat{x} & \geq\left(a-b X^{*}\right) x^{*}-\gamma\left(x^{*}\right)^{2}+\eta x^{*}
\end{aligned}
$$

Since by assumption that the effort function is increasing (i.e., we always have $X \leq \frac{a}{2 b}$ ), this inequality cannot hold unless we have the following condition satisfies.

$$
a+\eta-[(N+1) b+2 \gamma] x<0, \text { for } x>\hat{x} .
$$

This inequality leads us to the following proposition.

Proposition 4. The socially optimal extraction of the common resource is efficient if there exists a sufficiently high extraction cost such that for $x>\hat{x}$, we have

$$
\gamma>\frac{a+\eta}{2 x}-\frac{(N+1) b}{2} .
$$

It should be noted that if the above condition of $\gamma$ does not hold, then the socially optimal effort will be inefficient, i.e., we will have $U(\hat{x}) \leq U\left(x^{*}\right)$ for $\hat{x}<x^{*}$. We can observe that the lower bound of the extraction cost will increase if there is a reduction in the number of agents, which means more agents participating in resource extraction easier for the social optimum to achieve efficiency. 
Suppose that Proposition 4 holds, meaning that the socially optimal extraction is always efficient. Thus, an interesting question is how to motivate individuals to move toward the social optimum. To answer this question, let $\Delta_{x}$ be the difference between $x^{*}$ and $\hat{x}$. Then, we have

$$
\begin{aligned}
\Delta_{x} & =\frac{a+\eta}{2(N b+\gamma)}-\frac{a+\eta}{(N+1) b+2 \gamma} \\
& =\frac{(a+\eta)(1-N) b}{2(N b+\gamma)[(N+1) b+2 \gamma]} .
\end{aligned}
$$

This leads us to the following proposition.

Proposition 5. The Nash equilibrium extraction is efficient if there exists parameters $a, \eta$ and $\gamma$ such that $\Delta_{x}=0$, where

$$
\Delta_{x}=\frac{(a+\eta)(1-N) b}{2(N b+\gamma)[(N+1) b+2 \gamma]}
$$

We observe that $\Delta_{x} \rightarrow 0$ as $a, \eta$ decrease and $\gamma$ increases, suggesting that it is essential for the policymaker to ensure a sufficiently high extraction cost and low agent ability in order to motivate individuals to move toward the socially optimal extraction. It should be noted that when $N=1$, we would have $\Delta_{x}=0$, which means that there is only one agent who consumes the common resource. Moreover, a more increase in the number of agents $N$, a less likely to achieve efficiency.

In the general case when agents are identical, the problem becomes much more complication. Let us recall the interior equilibrium solution (see Equation (15)) as follows:

$$
\mathbf{x}^{*}=\frac{1}{2(b+\gamma)}\left(\mathbf{I}-\alpha \mathbf{\Phi}_{1}\right)^{-1} \iota
$$

where, $\boldsymbol{\Phi}_{1}=2 \boldsymbol{\delta}(\mathbf{I}-\mathbf{K G})-b \boldsymbol{\Delta}$. By comparing with the social optimal effort (see Equation 20), we observe that $2 \Phi_{2}<\Phi_{1}$ and thus $\hat{\mathbf{x}}>\mathbf{x}^{*}$. This suggests that the socially optimal effort is always efficient when there is a presence of network structure. Since we know that the interior equilibrium is always inefficient, a question arises is how could we achieve the efficiency. By comparing the difference between the interior and the social optimum, we observe that the inefficiency is come from the externality from the peer effect and the network structure in the matrix $\boldsymbol{\Phi}$. However, since the social structure is given (i.e., it is fixed), it is interesting to check which social structure could give the highest and the lowest optimal payoff as the network varies. 


\section{$5 \quad$ Numerical analysis}

In this section, we consider 4 different network structures: no network, circle/loop, star and complete network (see Figure 1). An empty network is when no agent connects to any other agents. The circle/loop and complete network are symmetric networks where each agent in the circle links to two other agents near-by, and each agent in the complete links to every other agent. A star network is a network where one agent in the center links to all other agents. In this case, a complete graph is a most strongly connected network in which each agent is connected to every other agent (i.e., each node has $N-1$ number of direct links. It is important to note that the number of direct links will increase as the network size increases in the case of a complete network. In contrast, in the circle network, each agent always has two direct neighbors, and the number of direct links will not increase when there is an increase in network size. In the star network case, only the central agent has an increasing direct network connection as the network size increases, while other agents have only one direct neighbor.

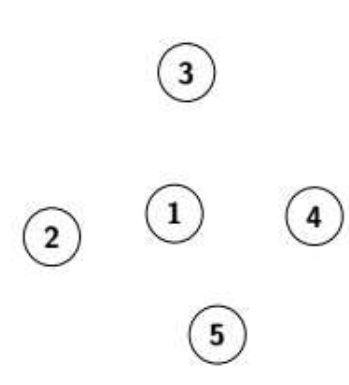

(a) No network

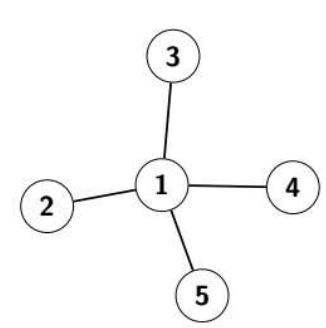

(b) Star

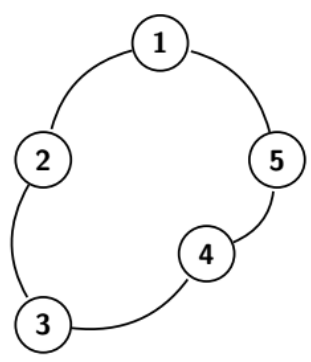

(c) Circle

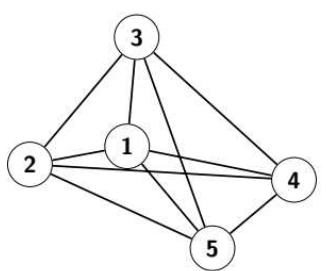

(d) Complete

Figure 1: The four different network structures.

We suppose that all the networks have 5 agents (i.e., a network of 5 agents). We consider that each agent can make an effort $x_{i}$ ranging from 0 to 1 , which are the $0 \%$ and $100 \%$ effort (i.e., $\left.x_{i} \in[0,1]\right)$. This means that the effort's capacity $\bar{x}=1$, i.e., no one can make an effort higher than effort's capacity of $100 \%$. Since by assumption that $X \leq \frac{a}{2 b}$, if we let $a=1$ for simplicity, then we choose $b=0.1$ because $b \leq \frac{a}{2 X}=0.1$. It should be noted that the parameter assumption of $b$ is also satisfied the condition $X \leq \frac{a}{b}$.

The ability parameter is uniformly distributed ranging from 0 to 0.5 . Thus, we have $E\left(\eta_{i}\right)=$ 0.25. For the extraction cost, we refer to the Proposition 4 in which $\gamma \geq \frac{a+E(\eta)}{2 x}-\frac{(N+1) b}{2}$ for $x>\hat{x}$. Thus, we choose $\gamma=0.8$ since from the previous condition, we have $\gamma>0.59$ for $x>0.5$. From the KKT condition (Case 2.a), the over-exploitation problem will happen if $\gamma \leq 0.325$. The comparison parameter also is uniformly distributed ranging from -0.2 to -0.1 for the case of assimilation in comparison $\left(\delta_{i}<0\right)$. Our strategy is to choose a low comparison parameter in the first step 
and then increase both comparison parameter to observe their impacts on agent's behavior. The parameter assumptions are summarized in Table 1.

Table 1: Parameter assumptions.

\section{Parameter values}

Agent $i$ 's effort: $x_{i} \in[0, \bar{x}]$ with $\bar{x}=1$.

Parameters of the effort function $f(X)=a X-b X^{2}: a=1$ and $b=0.1$.

Extraction cost parameter: $\gamma=0.8$.

Ability parameter: $\eta_{i}$ is uniformly distributed in $[0,0.5]$.

In the numerical simulation, we have $\boldsymbol{\eta}=(0.1437,0.3941,0.2044,0.4415,0.4702)$.

Assimilation comparison parameter: $\delta_{i}$ is uniformly distributed in $[-0.2,-0.1]$.

In the numerical simulation, we have $\boldsymbol{\delta}=(-0.1886,-0.1377,-0.1390,-0.1376,-0.1139)$.

Number of agents per network: $N=5$.

\subsection{Nash equilibrium level of effort}

We can calculate the interior equilibrium extraction effort for a given network structure from our parameter assumptions. Figure 2 shows the Nash equilibrium results for four different types of network (i.e., no network, circle, star, and complete network).

The results of the case without network in Figure 2 confirm our Proposition 1 that a higher ability agent would put more effort in extracting the common resource than a lower ability one. It should be noted that the matrix $\boldsymbol{\eta}=(0.1437,0.3941,0.2044,0.4415,0.4702)$ (see Table 1$)$ suggests that agent 1 is the one has lowest ability in the group, while agent 5 is the highest ability agent. Thus, agent 5 is the one who puts the highest effort into extracting the common resource.

In the presence of a network, the story becomes much more enjoyable since agents take their direct neighborhood extraction into account when making their decisions. The social comparison is assimilation in comparison, which means that agents try to imitate their direct neighbors' behaviors and make a decision as close as to the average direct neighborhood decisions. It should be noted that from the assumptions, we have $\boldsymbol{\delta}=(-0.1886,-0.1377,-0.1390,-0.1376,-0.1139)$ (see Table 1) which suggests that agent 1 who has lowest ability but (s)he is the one who cares most about his/her direct neighborhood behaviors. In contrast, agent 5 cares about his/her direct neighbors compared to other agents. Figure 2, the network plays a role in shaping the social comparison since 

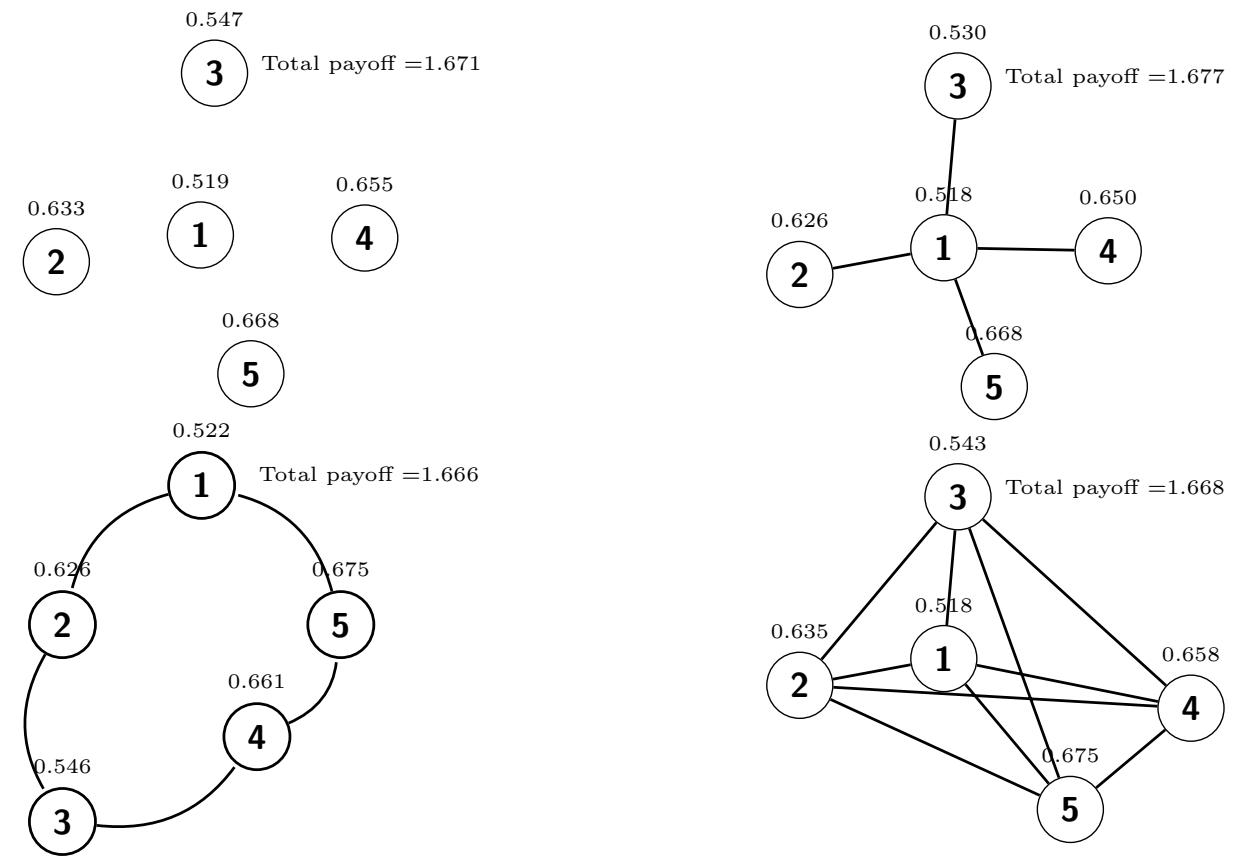

Figure 2: Nash equilibrium in 4 different networks.

there are differences in agents' behaviors and total payoff in different networks. To investigate the impact of social comparison and networks deeply, we let the comparison parameter vary and look at the equilibrium outcomes in different networks.

\subsection{Role of social comparison in network}

This section separates our discussions into two different cases: with/without an over-exploitation problem. In the first case, we consider that there is no over-exploitation with $\gamma=0.8$. In this case, agents would have no incentive to extract as much resource as possible because of the sufficiently high extraction cost. In the second case, we assume that there will be the over-exploitation of CPR with $\gamma=0.4$. In this case, without any invention (or policy), each agent would extract the CPR with the maximum effort's capacity $(\bar{x}=1)$. Figure 3 shows the average equilibrium extraction in the situation with/without over-exploitation problem.

Sub-figure (a) in Figure 3 shows that the assimilation in comparison cannot help to encourage the resource conservation as we expected when agents are in the decentralized networks (i.e., circle and complete network). This result can be justified by Proposition 3 because, in the decentralized network, we often have $\theta_{i}^{*} \rightarrow 0$ as $E\left(\delta_{i}\right)$ increases. As a result, a more increasing in $E\left(\delta_{i}\right)$, a more likely that there is an over-exploitation of the CPRs. However, in the centralized network (i.e., star network), agents seem to be motivated by reducing their extraction as the effected assimilation effect $E\left(\delta_{i}\right)$ increases because agent 1 who is the center of the star has the lowest ability $\eta$ and thus $\theta_{i}^{*}=x^{*}-\bar{x}_{N_{i}(g)}^{*}>0$ (see Proposition 3). 


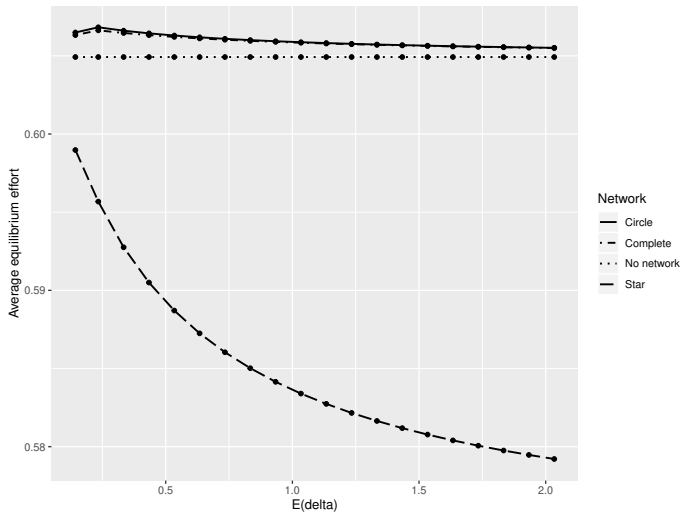

(a) $\gamma=0.8$

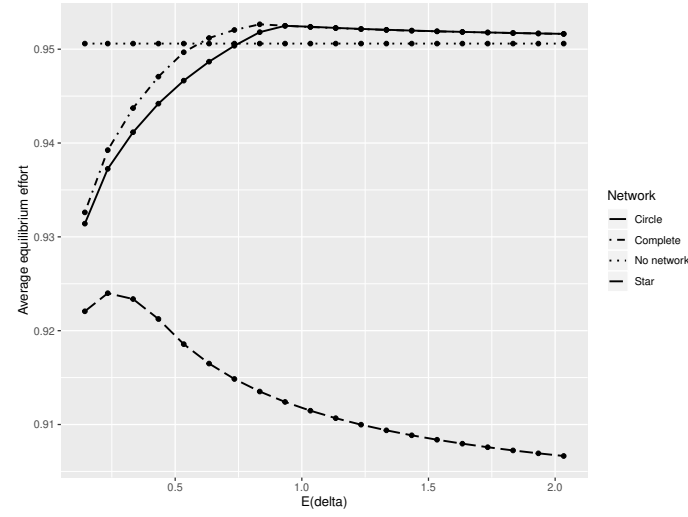

(b) $\gamma=0.4$

Figure 3: Average Nash equilibrium extraction in different networks.

As previously mentioned, in the case of no over-exploitation problem, it is unnecessary to introduce the social comparison to motivate the resource conservation because there is a sufficiently high extraction cost. Thus, it would be more interesting to investigate the role of social comparison in the case of over-exploitation. In the case of over-exploitation of the CPRs, all agents would put maximum effort into extracting the resource. By setting the extraction cost $\gamma=0.4$, we observe that in case of no social comparison, the average extraction equals 0.9505 (i.e., agents put $95 \%$ of their effort's capacity in extracting the resource). It should be noted that the maximum effort's capacity is each agent choosing $x=1.0$ (i.e., $100 \%$ effort). We observe that there is a possibility to encourage resource conservation with a relatively small $E\left(\delta_{i}\right)$. However, the over-exploitation problem can be becoming worse in the decentralized networks as $E(\delta)$ increases (see Sub-figure (b) in Figure 3). This result is because we always have in assimilation in comparison the property that $\theta_{i}^{*} \rightarrow 0$ as $E\left(\delta_{i}\right)$ increases. Therefore, a good strategy is to keep the comparison parameter as small as possible to encourage common resource conservation.

By comparing the circle and complete network, we observe that a network with more connections (i.e., complete network) performs slightly worse than the one with fewer connections in encouraging the conservation of the CPRs. This result is straightforward because, in a complete network, all agents are linked together. Thus they are more likely to mitigate others' behaviors than the circle network where agents compare their behaviors to only two direct neighbors.

It is important to note that both circle and complete network are decentralized networks and each agent in these networks has the same number of connections. Simultaneously, the star is a centralized network representing a real situation in which one central agent (i.e., a leader) plays a crucial role in maintaining the connections among agents in the network. We observe that the star network results in the highest total payoff with a lower level of extraction than other network types. It is because every agent in the star connects to only the center, and the central agent is the one who 
has the lowest ability in extracting the common resource. In our simulation, the center of the star has the lowest ability and thus puts the lowest effort into extracting the common resource. Thus, this leads to reduced resource extraction of the whole network. On the other hand, if we assume that the center in the star network is the one with the highest ability, then the over-exploitation problem will be more likely to happen (Figure 4 in Appendix B). Therefore, it is interesting for future studies to investigate in-depth using critical players in the network to encourage individual behavioral change toward environmental sustainability [3, 19].

\section{Conclusion and discussion}

Our study investigates the role of assimilation in social comparison in the network-based common pool resource game. We assume that agents are in a specific network, and they take their direct neighborhood actions into account when making their decisions (i.e., social comparison in networks). We suppose that there is assimilation in comparison which means that one changes their behaviors to minimize the difference/distance between them and others. We find that in the case of an empty network (i.e., no links between agents), a sufficiently high extraction cost is necessary to avoid the over-exploitation of a common pool resource. It is because no agents will take any other behaviors into their decision-making processes. Thus, as the cost of extracting the resource reduces, the tragedy of common is more likely to happen since all agents would have an incentive to put as much as possible their efforts to extract the common good.

In the presence of networks, over-exploitation is more likely to happen when there is a presence of assimilation in comparison. It is because agents are heterogeneous in their ability to extract the common resource (i.e., with the same amount of effort invested in extracting the CPR, one who has higher ability could earn higher benefit than others), a network with more connections would lead to a more severe exploitation problem than the one with fewer connections. This result is because the more the agents are linked to each other, the more likely they are to mitigate others' behaviors. As a result, agents would have a higher possibility to extract more resources if they observe that their neighbors or friends are doing so.

However, it is possible that the assimilation in social comparison can help to shift individual behaviors to promote the conservation of the CPRs. For instance, when the assimilation effect is low, we could encourage individuals to connect in a circle network since higher ability agents could also reduce their efforts when observing their neighbors extracting less resource. When the assimilation effect is high, we could encourage individuals to connect in a star network and incentivize the resource conservation to the central agent of a star network to motivate them towards a more sustainable behavior. Therefore, we suggest that policymakers should carefully 
consider the different types of network structure into account before implementing any policy (or intervention) that promotes the assimilative comparison.

Moreover, our results also suggest that when the assimilation effect is high, promoting the assimilation in the centralized networks (like a star network) is better than the decentralized one (like a circle or a complete network) in encouraging resource conservation. More specifically, we can promote the social comparison in the decentralized network, but it is essential to motivate the resource conservation of the central agent. Therefore, it is also crucial to provide information about how vital resource conservation is to the key player in the network (e.g., a central player in the star network) to incentivize resource conservation more effectively. However, as previously mentioned, how effective the social comparison in promoting the CPR conservation in the star network depends on the central agent's performance or ability, but identifying a key player (e.g., a central player in the star network) will not be easy if we do not observe the network structure. In reality, the key player could be the one who has a high degree of centrality, strong influence (e.g., influential, village leaders, etc.), first movers (e.g., seed farmers or block leaders), or gossiper, etc.

This analysis has several shortcomings which could be addressed by future research. Firstly, in this study, we only consider the exogenous network in which agents cannot choose their neighbors in the CPR game. An endogenous network of the CPR game could also be interesting to study. With the endogenous network, we can either use the static framework with the simultaneous move game in which agents choose their effort and the links simultaneously or the dynamic game in which agents first choose their neighbors and then the optimal effort. Secondly, we do not explore the possibility of the contrast in comparison which agents have incentives to differentiate with their neighbors because no agent is willing to differentiate with others by extracting less the common resource since there is a "substractability" of the commons. However, the contrast in comparison will be interesting to study if one can justify that there exists the case "doing worse, but feeling better" in extracting the common pool resource [13, 25]. Therefore, it is interesting that future research could consider this issue.

\section{Author Declarations}

\section{Funding and competing financial interests}

The author declares no competing financial interests.

\section{Data and code availability}

The statistical codes (in R software) used in this study are available from the author upon request. 


\section{Author contribution}

The author confirms sole responsibility for the following: study conception, data and analysis, interpretation of results, and manuscript preparation.

\section{References}

[1] Arrow, K. (1970). Political and Economic Evaluation of Social Effects and Externalities. In The Analysis of Public Output, Chapter 1, pp. 1-30. National Bureau of Economic Research.

[2] Barnes-Mauthe, M., S. Arita, S. Allen, S. Gray, and P. Leung (2013). The influence of ethnic diversity on social network structure in a common-pool resource system: Implications for collaborative management. Ecology and Society 18(1), 23.

[3] Borgatti, S. P. (2006). Identifying sets of key players in a social network. Computational $\&$ Mathematical Organization Theory 12(1), 21-34.

[4] Bromley, D. W. and M. M. Cernea (1989). The Management of Common Property Natural Resources: Some Conceptual and Operational Fallacies. World Bank Publications.

[5] Burkart, J. M., E. Fehr, C. Efferson, and C. P. van Schaik (2007). Other-regarding preferences in a non-human primate: Common marmosets provision food altruistically. Proceedings of the National Academy of Sciences 104(50), 19762-19766.

[6] Cárdenas, J. C., C. Mantilla, and R. Sethi (2015). Stable sampling equilibrium in common pool resource games. Games 6(3), 299-317.

[7] Frey, B. S. and S. Meier (2004). Social comparisons and pro-social behavior: Testing "conditional cooperation" in a field experiment. American Economic Review 94(5), 1717-1722.

[8] Frija, A., A. Zaatra, I. Frija, and H. AbdelHafidh (2017). Mapping social networks for performance evaluation of irrigation water management in dry areas. Environmental Modeling $\mathcal{E}$ Assessment 22(2), 147-158.

[9] Gelcich, S., T. P. Hughes, P. Olsson, C. Folke, O. Defeo, M. Fernández, S. Foale, L. H. Gunderson, C. Rodríguez-Sickert, M. Scheffer, et al. (2010). Navigating transformations in governance of Chilean marine coastal resources. Proceedings of the National Academy of Sciences 107(39), 16794-16799.

[10] Hardin, G. (1968). The tragedy of the commons. Science 162(3859), 1243-1248. 
[11] Isaac, R. M. and J. M. Walker (1988). Group size effects in public goods provision: The voluntary contributions mechanism. Quarterly Journal of Economics 103(1), 179-199.

[12] Kühnen, U. and B. Hannover (2000). Assimilation and contrast in social comparisons as a consequence of self-construal activation. European Journal of Social Psychology 30(6), 799-811.

[13] Lockwood, P., D. Dolderman, P. Sadler, and E. Gerchak (2004). Feeling better about doing worse: Social comparisons within romantic relationships. Journal of Personality and Social Psychology 87(1), 80.

[14] Mantilla, C. (2015). Communication networks in common-pool resource games: Field experimental evidence. Journal of Economic Behavior $\& 3$ Organization 118, 215-226.

[15] Mussweiler, T., K. Rüter, and K. Epstude (2004). The ups and downs of social comparison: Mechanisms of assimilation and contrast. Journal of Personality and Social Psychology 87(6), 832.

[16] Ostrom, E. (1990). Governing the Commons: The Evolution of Institutions for Collective Action. Political Economy of Institutions and Decisions. Cambridge University Press.

[17] Ostrom, E. (2008). The challenge of common-pool resources. Environment: Science and Policy for Sustainable Development 50(4), 8-21.

[18] Ostrom, E., R. Gardner, and J. Walker (1994). Rules, Games, and Common-pool Resources. University of Michigan Press.

[19] Patacchini, E. and Y. Zenou (2009). Juvenile delinquency and conformism. The Journal of Law, Economics, \& Organization 28(1), 1-31.

[20] Pearce, D. W. and R. K. Turner (1990). Economics of Natural Resources and the Environment. Johns Hopkins University Press.

[21] Poteete, A. R. and E. Ostrom (2004). Heterogeneity, group size and collective action: The role of institutions in forest management. Development and Change 35(3), 435-461.

[22] Rustagi, D., S. Engel, and M. Kosfeld (2010). Conditional cooperation and costly monitoring explain success in forest commons management. Science 330(6006), 961-965.

[23] Schweitzer, F., G. Fagiolo, D. Sornette, F. Vega-Redondo, A. Vespignani, and D. R. White (2009). Economic networks: The new challenges. Science 325(5939), 422-425.

[24] Sethi, R. and E. Somanathan (1996). The evolution of social norms in common property resource use. American Economic Review 86(4), 766-788. 
[25] Toma, C. L. (2013). Feeling better but doing worse: Effects of Facebook self-presentation on implicit self-esteem and cognitive task performance. Media Psychology 16(2), 199-220.

[26] Van Oel, P., D. Mulatu, V. Odongo, D. Willy, and A. Van der Veen (2019). Using data on social influence and collective action for parameterizing a geographically-explicit agent-based model for the diffusion of soil conservation efforts. Environmental Modeling \& Assessment 24(1), 1-19.

[27] Walker, J. M., R. Gardner, and E. Ostrom (1990). Rent dissipation in a limited-access common-pool resource: Experimental evidence. Journal of Environmental Economics and Management 19(3), 203-211.

\section{Appendix A}

\section{Lemma 1 - Sufficient condition of optimality}

From the necessary condition from Equation (13), we have

$$
\frac{\partial U_{i}}{\partial x_{i}}=f^{\prime}(X) \frac{x_{i}}{X}+f(X) \frac{x_{-i}}{X^{2}}-2 \gamma x_{i}+\eta_{i}+\frac{\partial I_{i}\left(x_{i}\right)}{\partial x_{i}} .
$$

By taking the derivative of the above equation with respect to $x_{i}$, we have

$$
\begin{aligned}
\frac{\partial^{2} U_{i}}{\partial x_{i}^{2}} & =f^{\prime \prime}(X) \frac{x_{i}}{X}+f^{\prime}(X)\left\{\frac{X-x_{i}}{X^{2}}\right\}+f^{\prime}(X) \frac{x_{-i}}{X^{2}}-f(X)\left\{\frac{2 x_{-i} X}{X^{4}}\right\}-2 \gamma+\frac{\partial^{2} I_{i}\left(x_{i}\right)}{\partial x_{i}^{2}} \\
& =f^{\prime \prime}(X) \frac{x_{i}}{X}+f^{\prime}(X)\left\{\frac{2 x_{-i}}{X^{2}}\right\}-f(X)\left\{\frac{2 x_{-i}}{X^{3}}\right\}-2 \gamma+\frac{\partial^{2} I_{i}\left(x_{i}\right)}{\partial x_{i}^{2}} \\
& =f^{\prime \prime}(X) \frac{x_{i}}{X}+\left(f^{\prime}(X)-\frac{f(X)}{X}\right)\left\{\frac{2 x_{-i}}{X^{2}}\right\}-2 \gamma+\frac{\partial^{2} I_{i}\left(x_{i}\right)}{\partial x_{i}^{2}} \\
& =\frac{1}{X^{2}}\left\{f^{\prime \prime}(X) X x_{i}+2 x_{-i}\left(f^{\prime}(X)-\frac{f(X)}{X}\right)\right\}-2 \gamma+\frac{\partial^{2} I_{i}\left(x_{i}\right)}{\partial x_{i}^{2}} .
\end{aligned}
$$

\section{Proposition 3 - Over-exploitation of the CPRs.}

Let us recall Equation (17) such that:

$$
f\left(X^{*}\right) \frac{x_{i}^{*}}{X^{*}}-\gamma x_{i}^{* 2}+\eta_{i} x_{i}^{*}+\delta_{i}\left(x_{i}^{*}-\bar{x}_{N_{i}(g)}^{*}\right)^{2} \geq f(\bar{X}) \frac{\bar{x}_{i}^{c}}{\bar{X}}-\gamma\left(\bar{x}_{i}^{c}\right)^{2}+\eta_{i} \bar{x}_{i}^{c} .
$$

We denote that $\theta_{i}^{*}=x_{i}^{*}-\bar{x}_{N_{i}(g)}^{*}$. Since the functional form of $f(X)$ is known, and for any $X \neq 0$, we thus have

$$
\begin{aligned}
\left(a-b X^{*}\right) x_{i}^{*}-\gamma x_{i}^{* 2}+\eta_{i} x_{i}^{*}+\delta_{i}\left(\theta_{i}^{*}\right)^{2} & \geq(a-b \bar{X}) \bar{x}-\gamma \bar{x}^{2}+\eta_{i} \bar{x} \\
\gamma\left(\bar{x}^{2}-x_{i}^{* 2}\right) & \geq\left(a+\eta_{i}\right)\left(\bar{x}-x_{i}^{*}\right)+b\left(X^{*} x_{i}^{*}-N \bar{x}^{2}\right)-\delta_{i}\left(\theta_{i}^{*}\right)^{2} .
\end{aligned}
$$

Since we know that $\bar{x}^{2}-x_{i}^{* 2}=\left(\bar{x}-x_{i}^{*}\right)\left(\bar{x}+x_{i}^{*}\right)$, we therefore would have

$$
\gamma \geq \frac{a+\eta_{i}}{x_{i}^{*}+\bar{x}}+\frac{\delta_{i} \theta_{i}^{* 2}-b\left(X^{*} x_{i}^{*}-N \bar{x}\right)}{\left(x_{i}^{*}-\bar{x}\right)\left(x_{i}^{*}+\bar{x}\right)} \equiv \underline{\gamma}_{i}
$$




\section{Appendix B}

Nash equilibrium extraction with different ability parameters

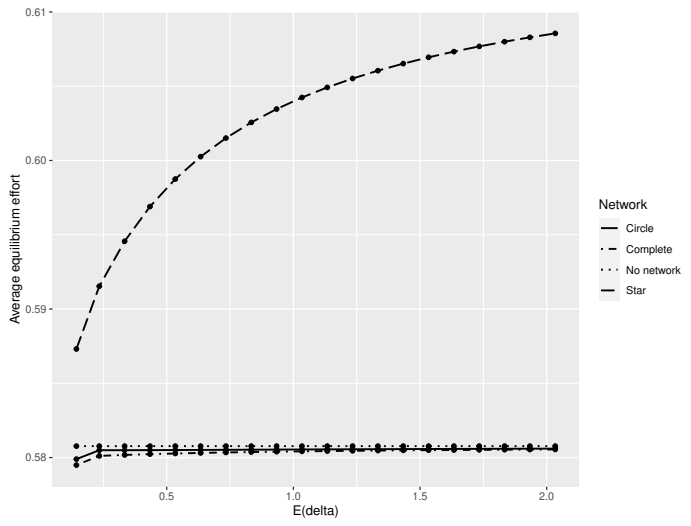

(a) $\gamma=0.8$

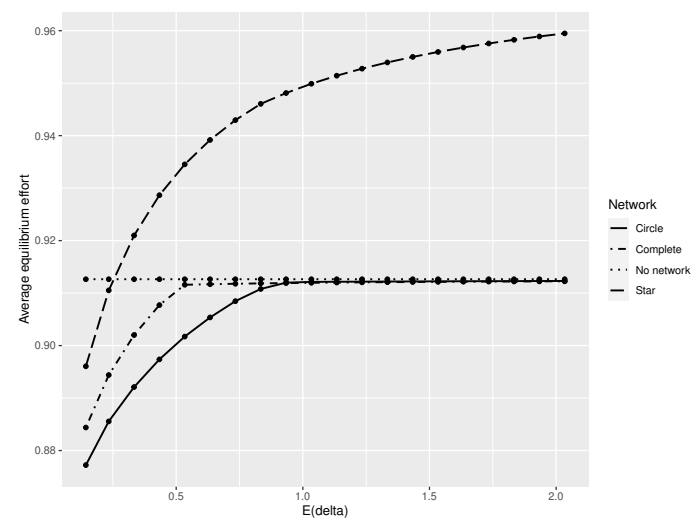

(b) $\gamma=0.4$

Figure 4: Average Nash equilibrium extraction in different networks with $\boldsymbol{\eta}=$ $(0.477,0.468,0.119,0.127,0.195)$. 
Figures

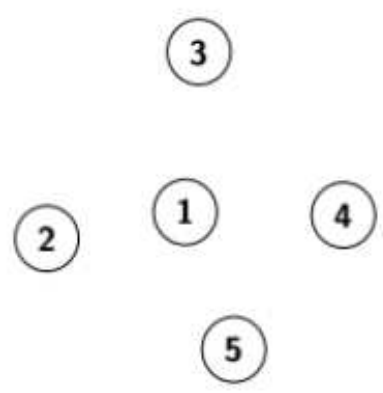

(a) No network

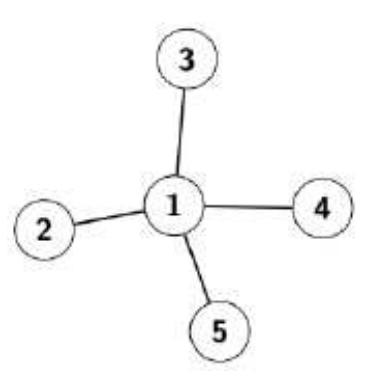

(b) Star

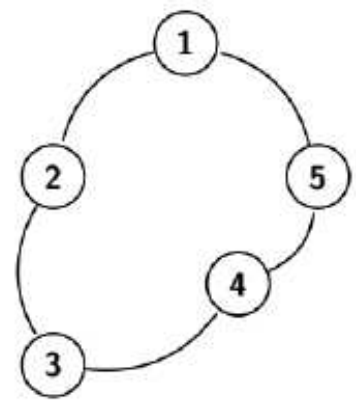

(c) Circle

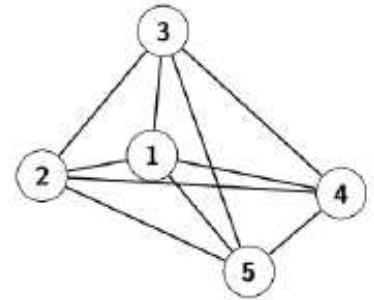

(d) Complete

\section{Figure 1}

The four different network structures.
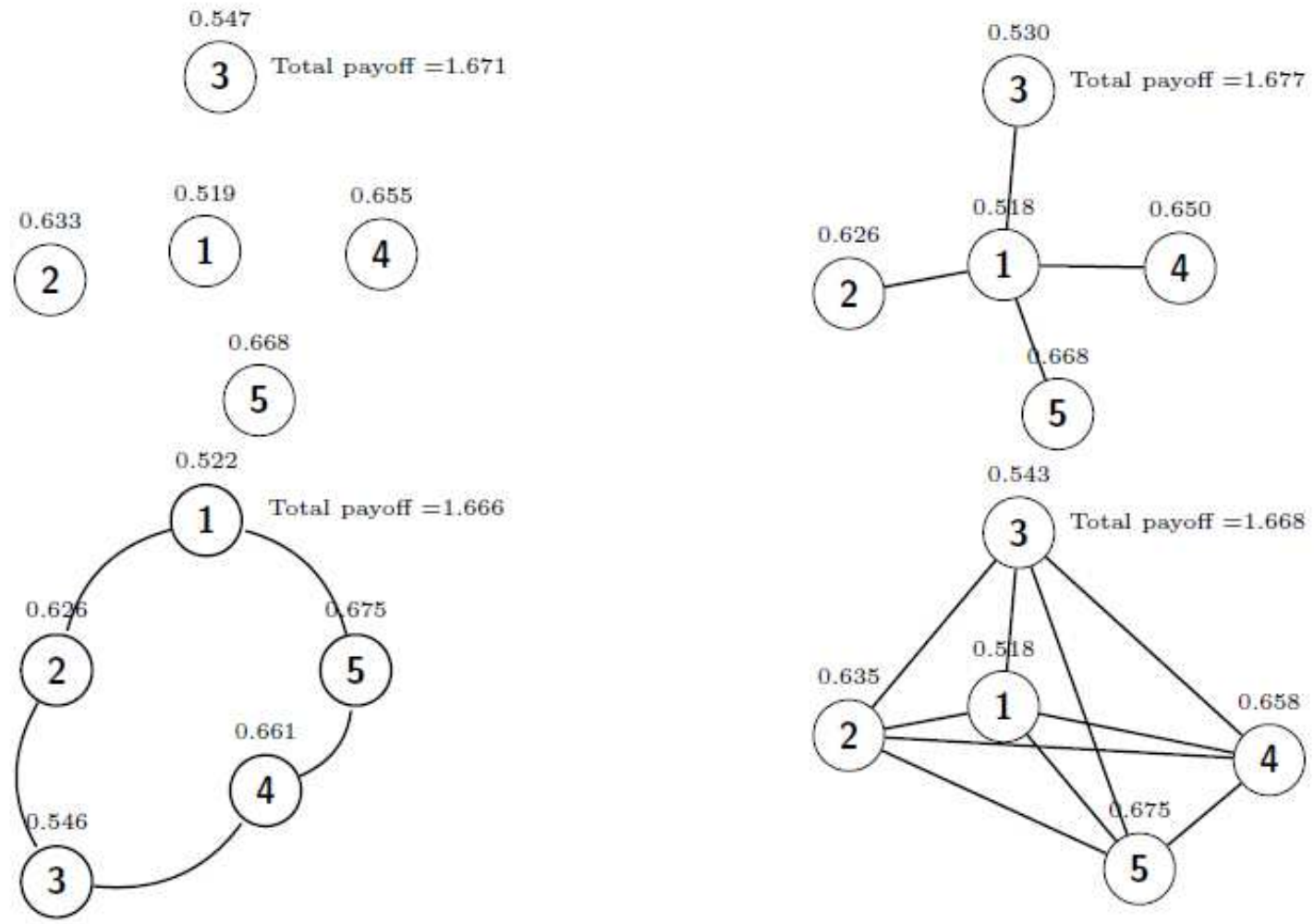

\section{Figure 2}

Nash equilibrium in 4 different networks. 


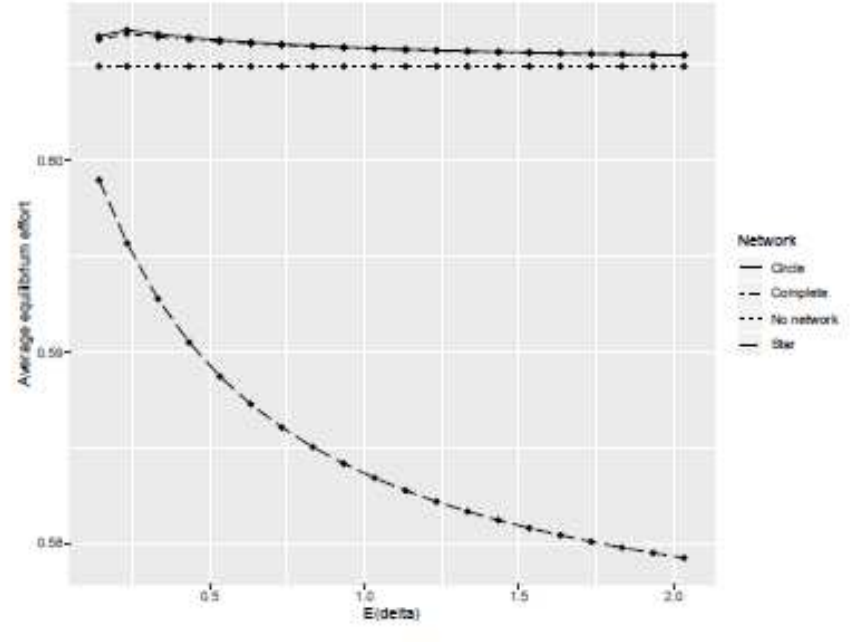

(a) $\gamma=0.8$

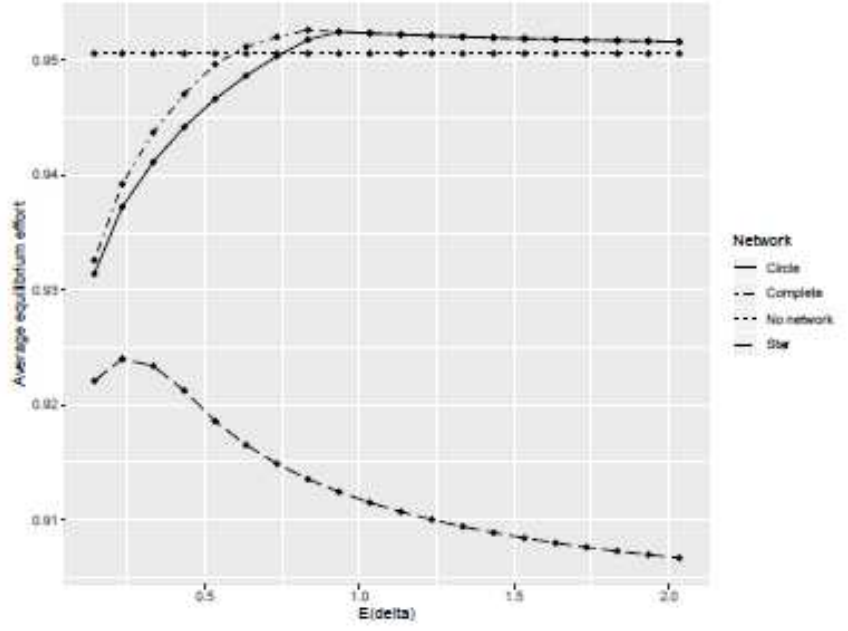

(b) $\gamma=0.4$

Figure 3

Average Nash equilibrium extraction in different networks. 